\title{
La prévention - notamment contre le VIH - commence au cabinet médical
}

A l'heure actuelle, on accorde une grande importance à la prévention dans de nombreux domaines. La prévention des problèmes de santé implique la connaissance des facteurs déclenchants d'une maladie. En effet, ce sont les médecins qui, les premiers, ont soulevé le problème du tabagisme. Les conséquences de la surcharge pondérale et du manque d'exercice ont également été abordées au cabinet médical. Nous autres médecins disposons d'une grande expérience, car plus que nul autre groupe professionnel, nous sommes confrontés à une foule de destins individuels. Le fait que nous soyons personnellement impliqués nous incite à nous engager en faveur de la prévention dans notre domaine. L'ophtalmologue, par exemple, s'engagera en faveur de la reconnaissance précoce du glaucome, le pneumologue en faveur de la prévention du tabagisme, etc. Pour ce qui est des spécialistes en médecine interne ou des généralistes, ils sont quotidiennement concernés par la plupart des domaines de la prévention. Jour après jour, de nombreux médecins s'engagent activement en faveur de la prévention sans en tirer de gloire personnelle.

Dans le milieu des années 80, alors que l'épidémie du sida se déclarait en Suisse, des spécialistes du VIH se sont battus dès la première heure par le biais d'exposés, de communiqués de presse et de concepts de prévention. Depuis le début, ces derniers se sont impliqués pour qu'un entretien ait lieu simultanément aux tests de dépistage du VIH. Ils se sont engagés et s'engagent aujourd'hui encore, motivés en cela par leur expérience personnelle au contact des personnes infectées par le virus du sida. Or, par expérience, nous savons que trop souvent encore, c'est un manque d'informations et un comportement erroné qui mènent à l'infection. En outre, nous constatons aussi fréquemment une mauvaise interprétation des tests négatifs.
Les données de l'enquête suisse sur la santé et de l'Office fédéral de la santé publique montrent qu'en Suisse, 300000 tests VIH sont effectués chaque année hors transfusions sanguines, dont une grande partie dans les cabinets médicaux. Le test VIH est l'occasion idéale pour mener un bref entretien sur la prévention. Même si sa durée n'est que de trois minutes toutes les trois consultations, ce sont 5000 heures investies annuellement dans le conseil. Sur le plan financier, le temps consacré équivaudrait à un montant bien supérieur à celui dont dispose l'OFSP pour toutes ses activités de prévention VIH/sida.

Conscients de l'importance que revêt ce travail, nous autres médecins veillons aussi à ce qu'il soit réalisé de manière professionnelle. Le Bulletin de l'OFSP de ce mois de juillet contient les résultats d'une enquête sur les conseils donnés au cabinet médical concernant le test VIH. Ces résultats sont réjouissants et encourageants car ils révèlent qu'un nombre élevé de médecins sont conscients de l'importance de leurs activités de prévention VIH mais aussi que ce conseil peut encore être optimisé à bien des égards.

Afin d'optimiser le conseil de prévention, la Commission clinique et thérapie VIH/sida a rédigé un petit guide pour les cabinets médicaux, également publié dans le Bulletin de l'OFSP et sous forme abrégée dans le présent numéro. Ce guide présente des suggestions sur la manière de mener un entretien de conseil avec le patient. Il contient en outre les principaux éléments de la prévention VIH afin qu'on puisse continuer à les communiquer de manière uniforme. N'oubliez pas de consulter ce guide lorsque vous effectuerez le prochain test VIH et agissez de sorte que la prévention au cabinet médical conserve toute son importance!

Pietro Vernazza, président de la Commission clinique et thérapie VIH/sida, St-Gall 